\title{
La historia en la basura: los archivos perdidos de Donald Pierson*
}

\author{
Roberto Lima
}

\begin{abstract}
En este texto se reconstruye e interpreta el proceso que culminó con la destrucción del archivo de datos de campo que el equipo de Donald Pierson (1900-1995) recolectó en su investigación sobre el Río San Francisco. Dicho archivo consistía en varios millares de fichas mecanografiadas, con información recogida por el equipo de 22 investigadores que Pierson coordinó, en una investigación que incluyó diez ciudades dispersas por todo el Valle del Río San Francisco en la década de 1950. Se analiza, entre otros factores, la indiferencia de la entidad que tenía la custodia del acervo, la subestimación de este autor en la historiografía de la antropología brasileña, estrechamente ligada a las críticas a los estudios de las comunidades, y la vulnerabilidad de los archivos brasileños.
\end{abstract}

Palabras Clave: Donald Pierson, Río San Francisco, archivos de antropología, notas de investigación, destrucción de archivos

\section{History in Trash: the Lost Archives of Donald Pierson}

This paper reconstructs and interprets the process that culminated with the destruction of field data archive that the team of Donald Pierson (1900-1995) collected in its investigation on San Francisco River. This archive consisted of several thousands of typed cards, with information collected by 22 researchers who were coordinated by Pierson on a research that included ten cities placed throughout San Francisco River Valley in the 1950's. Among other aspects, it discuses the disregard of the entity that held the archive in custody, the underestimation of the author in the historiography of Brazilian Anthropology, closely linked to the criticism of community studies, and the vulnerability of Brazilian archives.

KEYWORDS: Donald Pierson, San Francisco River, Anthropology archives, research notes, archives destruction

\author{
RoBERTO LIMA: Universidad Federal de Goiás, Goiânia, Brasil \\ nadanacuca@gmail.com \\ Traducción: Clara Isabel Martínez
}

Desacatos, núm. 34, septiembre-diciembre 2010, pp. 107-118

Recepción: 30 de agosto de 2008 / Aceptación: 13 de mayo de 2009

* Agradezco al Acervo Memoria de la Compañía de Desarrollo de los Valles de San Francisco y del Parnaíba (CODEVASF) y al Archivo Edgard Leuenroth de la Universidad Estadual de Campinas, São Paulo, el acceso a los manuscritos de Pierson aquí citados, especialmente a la Biblioteca Pública Luiz de Bessa de Belo Horizonte, Minas Gerais, por la oportunidad de investigar en lo que quedó de los ficheros del proyecto San Francisco. Anexamos a este texto la moción de repudio aprobada en la plenaria general de la XXII Reunión de la Asociación Brasileña de Antropología (ABA), el 19 de julio de 2000. La parte inicial de esa investigación fue financiada con una beca de doctorado de la CAPES y del CNPq. La redacción final tuvo el apoyo del CNPq-Brasil (beca PDE). Agradezco las sugerencias de los colegas, mismas que fueron incorporadas dentro de mis límites y de los límites de extensión del texto. Obviamente, todo lo que está escrito aquí es de mi entera responsabilidad. 
$\mathrm{E}$ 1 presente artículo trata de hechos ocurridos en la época en que estaba investigando para mi tesis de doctorado sobre el Río San Francisco (defendida en el año 2002). El objetivo es presentar una interpretación de lo sucedido a partir de la información recolectada. Al buscar fuentes bibliográficas sobre ese río, cuya posición en el imaginario nacional es la de un ideologema ${ }^{1}$ fundamental en la creación del discurso nacionalista brasileño, entré en contacto con los trabajos de Donald Pierson (1900-1995). Inspirado por la propuesta que dicho autor expresó en varias ocasiones — de que se hicieran reanálisis del material bruto de campo del proyecto de investigación que él había coordinado en la década de 1950 (y que posteriormente él mismo donó a la Fundación João Pinheiro)—, elaboré como primera propuesta de tesis una actualización de los datos sobre prácticas religiosas recogidos en las diez ciudades estudiadas por Pierson y su equipo. Como veremos más adelante, esto no fue posible.

¿Acaso esta historia tendría que comenzar por el fin? Si es así, pues que el principio sea a través de la pista que detonó esta crisis: una carta de una investigadora de la Universidad de Duke en la cual se afirma que los archivos de Donald Pierson y sus colaboradores, referentes a su investigación en el Río San Francisco, habían sido tirados a la basura ${ }^{2}$.

¿Tienes alguna información sobre el contenido de los archivos de Pierson en Belo Horizonte o en Campinas? Tengo una amiga en Brasil que llamó a Belo [Horizonte] (Funda-

\footnotetext{
${ }^{1}$ El término ideologema lo tomamos de Jameson, quien señala: "is an amphibious formation, whose essential structural characteristic may be described as its possibility to manifest itself either as a pseudoidea - a conceptual or belief system, an abstract value, an opinión or prejudice - or as a protonarrative, a kind of ultimate class fantasy about the 'collective characters' which are the classes in opposition" (Dameson, 1981: 87, subrayado desde el original). El Río San Francisco ha sido llamado "mediterráneo brasileño", "río de la integración nacional", "factor precipuo de la existencia brasileña", entre otros epítetos.

2 Pierson era un autor prolífico. Además del archivo destruido de la Fundación João Pinheiro, también existen partes de sus archivos personales (cartas, originales y manuscritos) en el archivo Edgard Leuenroth, Universidad de Campinas (67 fólderes de archivo), como parte del acervo del bello proyecto História da Antropologia do Brasil, dirigido por Mariza Corrêa, y en los "Donald Pierson Papers" de las Special Collections de la biblioteca de la Universidad de Florida, donde además existen "4 pies lineales" de originales de Pierson.
}

ción João Pinheiro) y le avisaron que casi todo el archivo de Pierson había sido desechado. Estoy esperando más detalles de esto y en cuanto los tenga te aviso. ¿Ya consultaste el archivo en Belo [Horizonte]?

Así comienza este relato cuyo origen se puede ubicar el 28 de diciembre de 1951, cuando Donald Pierson, representando a la Escuela de Sociología y Política de São Paulo y a la Smithsonian Institution, firmó un convenio con la Comisión del Valle de São Francisco (CVSF), por un valor de 490000 cruzeiros $^{4}$ para realizar "estudios de carácter sociológico y ecológico de la región” (Peltier y Pierson, 1951: 85) ${ }^{5}$. De allí resultó el proyecto en el que Donald Pierson y más de dos decenas de colaboradores ${ }^{6}$ realizaron

\footnotetext{
${ }^{3}$ Como no solicité autorización a la investigadora para la publicación de su carta, conservaré el anonimato de la misma. Procederemos de igual manera con todas las cartas que me hicieron llegar "con carácter personal" y que estoy utilizando como material de investigación en este artículo.

${ }^{4}$ La actualización de ese valor es difícil por los años y la inflación. Por las tablas del Banco Central de Brasil, tendríamos el valor de R\$ 11774030 en mayo de 2010 o cerca de U\$ 65400 00. Pero ese valor parece más bien pequeño frente a lo necesario para el proyecto.

${ }^{5}$ La CVSF fue una agencia estatal creada el 15 de diciembre de 1948 para promover el desarrollo regional, teniendo como modelo a la Tennessee Valley Authority. No obstante, por diferentes motivos - entre ellos los relacionados con las vicisitudes de la política brasileña, por un lado, y por el otro, debido al propio carácter de sus dirigentes-, esa agencia nunca funcionó teniendo como referencia un plan de desarrollo regional, a pesar de contar con un presupuesto seguro ( $1 \%$ de lo recabado por la Unión). Por el contrario, sus acciones siempre se dieron como consecuencia de alianzas personales y con el eterno carácter de "paliativo" (Dantas, 1972). La enorme cantidad de irregularidades administrativas hizo que la agencia fuera rebautizada como Superintendencia del Valle de San Francisco (SUVALE) y posteriormente, en 1974, como Compañía de Desarrollo del Valle de San Francisco (CODEVASF). La conjunción presupuesto asegurado/desdén por un plan de acción ayuda a explicar algunos de los acontecimientos narrados en este artículo, como la demora en publicar los resultados del proyecto de Pierson.

${ }^{6}$ En total eran 22 colaboradores: Levy Cruz, Aparecida Joly Gouveia, Lucas Nogueira Garcez, Frederico Barros Brotero, Zilda Cruz (esposa de Levy Cruz), Fernando Altenfelder Silva, Maria Galvao Cardoso, Lídia Altenfelder Silva (esposa de Fernando), Cândido Procópio Camargo, Octavio Costa Eduardo, Plínio Figueiredo, Arthur de Moraes Cezar, Natália Rodrigues Bittencourt, Alceu Maynard Araújo, Maria Wagner Vieira da Cunha, João Vicente Cardenuto, Noêmia Pereira Toledo, Geraldo Semenjato, Alfonso Trujillo Ferrari, Esdras Borges Costa, Maria Isabel dos Santos, Gastao Thomas Almeida, Neide Carvalho, Padre Aldemar Moreira. Esta lista corresponde a los integrantes de los cinco equipos de investigación participantes en el proyecto. Debo decir que muchos de ellos ya murieron, lo que hace de la reconstitución del archivo un trabajo de gambusino entre parientes y archivos dispersos por el territorio brasileño.
} 
la principal investigación que se ha hecho hasta ahora sobre el Valle del Río San Francisco. La pesquisa se llevó a cabo en diez ciudades divididas en cinco pares, distribuidos de la siguiente manera: el primero en el "altiplano occidental", la ciudad de Correntina y la región al oeste de ésta, denominada Gerais; para el segundo se escogió un lugar ribereño de economía pesquera, Xique-Xique y Marrecas; el tercero en el área de Caatinga, donde hay cría de cabras y se estaba introduciendo el riego, Cabrobró e Isla de la Asunción; el cuarto fue en la región de cultivo de arroz en el bajo valle, Piassabucu y Potengi; el quinto y último fue en el alto San Francisco (Hacienda Diamantina). En términos de cobertura, este proyecto tal vez sólo pueda compararse con el proyecto Brasil Central, dirigido por Roberto Cardoso de Oliveira y David Maybury-Lewis.

Como resultado inmediato de esta investigación, varios libros y numerosos artículos fueron publicados ${ }^{7}$, pues en las cláusulas $8^{a}$ y $9^{a}$ del convenio constaba que:

La Escuela de Sociología y Política de São Paulo se obliga a entregar a la CVSF [...] las monografías de las comunidades estudiadas, que serán elaboradas por los respectivos directores de investigación, bajo la dirección general del profesor Donald Pierson, y que serán publicadas por la CVSF y [...así como] los originales de un libro a ser elaborado, en inglés, para el Institute of Social Anthropology de la Smithsonian Institution por el profesor Donald Pierson, sobre toda la región del Valle del Río San Francisco (Peltier y Pierson, 1951: 87 verso).

Pero también se obtuvo otro resultado — realizado en las sombras de la investigación- del cual no se habló en aquella época: como los 22 investigadores estaban obligados a entregar al coordinador del proyecto copias de sus notas de campo, éstas fueron clasificadas y mecanografiadas con copia, dando lugar a un archivo, contenido

\footnotetext{
${ }^{7}$ Cito sólo las monografías: Cerrado e Retiro, de Esdras Borges Costa, 1960; Xique-Xique e Marrecas, de Fernando Altenfelder Silva (1961); Canta Brasil (1957), Populacoes Ribeirinhas do Baixo São Francisco (1961), Escorco de Folclore de uma comunidade (1962), todos de Alceu Maynard de Araújo; Porengi de Alfonso Ferrari (1960); O Homem do Vale do São Francisco, de Pierson (1972). Gran parte de los artículos fue publicada en la revista Sociologia, dirigida en aquella época por Emilio Willens y Romano Barreto. Esta revista amerita una exhumación, pues en sus volúmenes se puede apreciar buena parte del movimiento de creación de los currículos de Ciencias Sociales en Brasil en el periodo que va de 1940 a 1960.
}

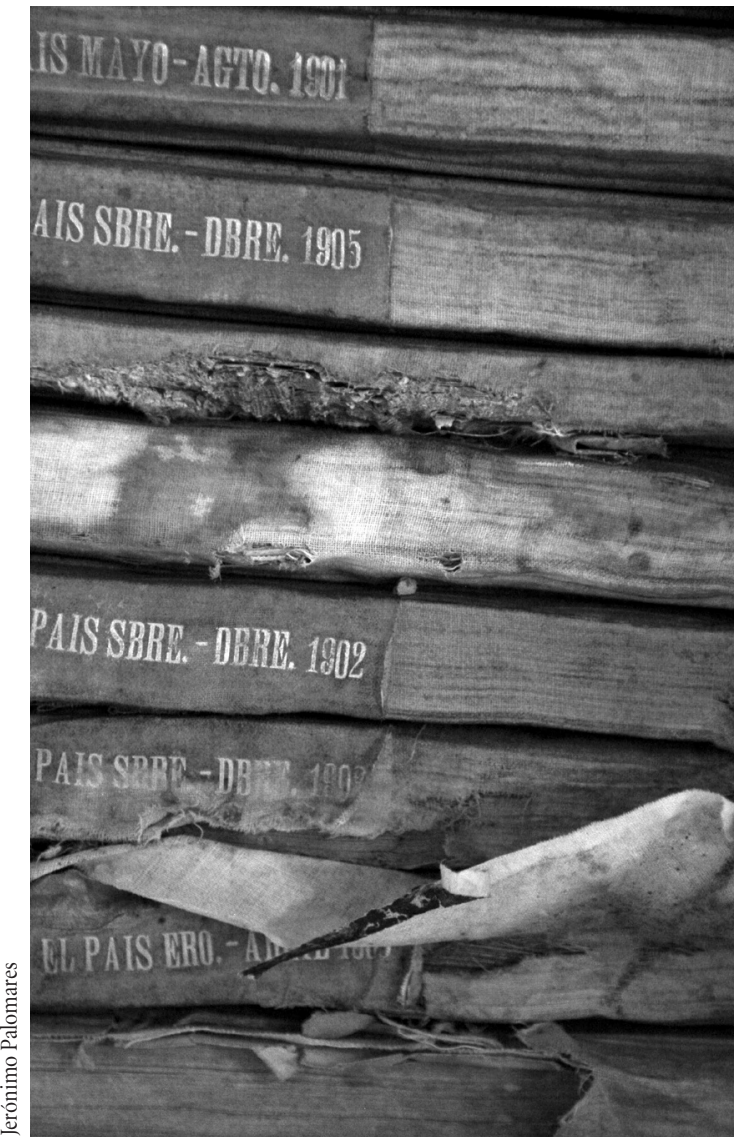

Biblioteca "Rafael García Granados”, 2010.

en 15 cajitas de $26 \times 18 \times 12.5$ (Pierson, 1987). En suma, un registro minucioso de la vida de la población ribereña en las puertas del inicio del ciclo de las grandes hidroeléctricas y de los distritos de riego ${ }^{8}$.

\footnotetext{
${ }^{8}$ Es importante subrayar el valor histórico de estas fechas, pues en 1953 se define el lugar de la primera gran hidroeléctrica en el Río San Francisco, la cual fue inaugurada en 1955, ejerciendo un profundo impacto no sólo en las comunidades que tuvieron que ser reubicadas, sino también en las comunidades de aguas abajo que vivían de la agricultura en tierras inundables (en Três Marias el río variaba su descarga entre $200 \mathrm{~m}^{3} / \mathrm{s}$ en la época de secas y $6.500 \mathrm{~m}^{3} / \mathrm{s}$ en las grandes inundaciones) y que vieron cómo el caudal del río se fue regularizando en un volumen aproximadamente constante de $550 \mathrm{~m}^{3} / \mathrm{s}$. Este fenómeno es parte del imaginario de la población ribereña, la comunidad cimarrón del Río das Rãs, donde los habitantes, al no darse más las grandes inundaciones, afirman que "el río comenzó a secarse con [la construcción de] Três Marias".
} 
Así es como a principios de la década de 1980, con más de 80 años de edad, Pierson comenzó a preocuparse por el destino que tendría aquel rico material cuando él muriera. Entonces retomó la correspondencia con cada uno de los investigadores que participaron en el proyecto, con el objetivo de solicitarles su autorización para donar las copias de las fichas que estaban en su poder" a una "institución de investigación idónea" que, según su criterio, tendría que ser brasileña, de preferencia ubicada en el Valle del Río São Francisco. Pierson estaba muy motivado por una práctica incipiente en esa época: el reanálisis antropológico:

La esperanza de encontrar un lugar seguro donde pudieran guardarse permanentemente mis copias de notas viene de lejos; sin embargo, este sentimiento se hizo más fuerte cuando, a solicitud de los directores de los Annals de la American Academy of Political and Social Science, elaboré la reseña de una obra del antropólogo Robert E. Hinshaw, quien al estudiar al pueblo localizado a los bordes del lago Atitlán, en Guatemala, utilizó con fines comparativos las notas de otro antropólogo, el profesor Sol Tax, referentes al mismo pueblo pero de 30 años atrás. Así, fue posible que Robert E. Hinshaw estudiara la vida de aquel pueblo in depth, o sea, a través de los cambios experimentados en las tres décadas previas a su investigación, obteniendo así, fácilmente, una visión pormenorizada de aquello que fue [el pueblo] treinta años atrás para aquello que es ahora (Pierson,1987: 107-108). (Cursivas mías) ${ }^{10}$

Pierson estaba consciente de que la preservación de este archivo mantendría una puerta abierta entre el pasado y el porvenir en las investigaciones antropológicas sobre la región. La búsqueda de un lugar adecuado y seguro fue bastante cuidadosa, como se desprende de una carta dirigida a Rodrigo Octavio Coutinho, fechada el 4 de agosto de 1981:

\footnotetext{
${ }^{9}$ Esta correspondencia, realizada entre 1981 y 1983, se encuentra en el Archivo Edgard Leuenroth, especialmente en los fólderes 002-008, 056-058, 063-070.

${ }^{10}$ Posteriormente este tipo de estudios se volvió bastante común. Se pueden citar los reanálisis del material de Tepoztlán de Robert Redfield hechos por Oscar Lewis, de las islas Trobriand de Bronislaw Malinowski por Stanley Tambiah, de los ndembu de Víctor Turner por Marisa Peirano y, para dar un ejemplo bien brasileño, de los krahó de Júlio Melatti por Manuela Carneiro da Cunha.
}

Actualmente mi preocupación tiene que ver con las notas de campo escritas por nuestros 22 investigadores y por mí en ese entonces, organizadas en São Paulo por categorías y mecanografiadas por Maria Isabel dos Santos, mi fiel secretaria en aquel tiempo, y sus auxiliares. No quiero que estas copias de notas desaparezcan un día, cuando yo ya no esté [...], todavía hay en ellas muchas y muchas cosas inéditas (Carta depositada en el Archivo Edgard Leuenroth).

En la correspondencia con Rodrigo Octavio Coutinho se percibe que este último también estaba gestionando la donación al Archivo Público Mineiro, a la Universidad Federal de Minas Gerais (UFMG) y a la Universidad de Brasilia (UnB). Al respecto se dice que la UFMG se mostró "poco comprensiva" (según palabras de Coutinho) y la UnB tampoco aceptó el material, como se desprende de la carta (5 de junio de 1983) de Pierson a Roberto Cardoso de Oliveira, entonces en la Universidad de Brasilia. Según dicha carta se infiere que Cardoso de Oliveira rechazó la custodia del material por no entender de qué se trataba (la carta de Pierson es una explicación de la naturaleza del acervo y no está escrita en el tono usualmente pulido y cordial del autor).

Finalmente, Pierson acordó con la Fundación João Pinheiro que fuera ésta la depositaria del acervo. En testimonio concedido a Mariza Corrêa (publicado en Corrêa, 1987) se aprecia claramente el orgullo que él sentía de esta fundación, por ser sus directores "brasileños de futuro" y "hombres de visión" y, para él, esta opinión se confirmaba con la carta del director de la Fundación João Pinheiro, Aluísio Pimenta:

Esta oportunidad mucho honra a la Fundación João Pinheiro, que viene procurando ofrecer su colaboración en el esfuerzo de preservar el acervo cultural... (carta de Pimenta a Pierson, cit. por Pierson, 1987) ${ }^{11}$.

A pesar de que Pierson hace una detallada descripción de los trámites para la donación, no proporciona la fecha exacta. No obstante, se puede decir, sin temor a equivocarnos, que la donación se hizo en 1984. Dada la proxi-

\footnotetext{
${ }^{11}$ Esta carta, fechada el 7 de octubre de 1983, se encuentra en el Archivo Edgard Leuenroth.
} 
midad de la fecha, se entiende el asombro y el horror que causó saber que en tan poco tiempo $80 \%$ de este material haya sido destruido, según cálculo aproximado presentado vía telefónica por la dirección de la Biblioteca Pública Estatal Luiz de Bessa, en Belo Horizonte, adonde el archivo fue transferido. En los primeros contactos, tanto el personal de la Fundación João Pinheiro como el de la Biblioteca Pública Estatal Luiz de Bessa fueron extremadamente evasivos: no se sabía por qué y se ignoraba la fecha; tampoco se conocían las circunstancias en que se había hecho la transferencia (de las conversaciones sólo se desprendía que, aparentemente, la destrucción ocurrió durante el traslado del material). Sobre estas cuestiones, la lacónica respuesta obtenida, tanto de la Fundación João Pinheiro como de la Biblioteca Pública, era: "sucedió en el gobierno pasado".

Fue necesario viajar a Belo Horizonte para empezar a entender lo que había sucedido. A la negativa inicial de la Fundación João Pinheiro y de la Biblioteca Pública Estatal Luiz de Bessa de explicar los hechos, siguieron las declaraciones, a veces dramáticas, de los directores de las dos instituciones ${ }^{12}$ :

\section{Sr. Roberto Lima:}

He intentado hablar por teléfono con usted varias veces sin conseguirlo. Quiero aclarar que el archivo de Donald Pierson estuvo en nuestra Biblioteca a lo largo de 12 años, yo misma lo recibí. Estuve fuera de la Fundación João Pinheiro durante siete años y cuando regresé consideré mejor transferirlo a la Colección Mineiriana en la Biblioteca Pública Estatal, [el mueble con] ese archivo se fue junto con otros archivadores vacíos que donamos a la Biblioteca. Lo sentimos, pero la funcionaria que los recibió, ignorando la importancia del acervo, a pesar de que el archivador estaba sellado y pegado con cinta adhesiva, todas las fichas fueron tiradas a la basura [sic]; otra funcionaria, al descubrir la falla y sabiendo de nuestra transacción y del contenido de los cajones, intentó recuperarlo, consiguiendo sólo el 10\% de lo enviado. Todo esto fue para nosotros motivo de gran insatisfacción y frustración, pues conocíamos la gran importancia del archivo; nos sentimos culpables de la pérdida y de la

\footnotetext{
${ }^{12}$ Como ya se explicó anteriormente, seguiré sin dar los nombres de las personas involucradas, básicamente por dos motivos: 1) por ser datos de investigación cuyos informantes solicitaron guardar la identidad, y 2) porque creo que la cuestión principal es institucional.
}

falla, sin saber dónde nos habíamos equivocado. Y por increíble que parezca, desde entonces varias personas nos han buscado para la consulta de tales fichas. Estoy a su entera disposición para cualquier otra aclaración.

En las declaraciones de las personas directamente involucradas, tanto de la Fundación João Pinheiro como de la Biblioteca Pública Luiz de Bessa, siempre se alegó la existencia de "atenuantes" para entender el porqué de la pérdida ocurrida en diciembre de 1998: había presión del secretario de Cultura para la inauguración del nuevo edificio de la biblioteca, y se consiguió la donación de cierto número de estantes para libros y ficheros con la Fundación João Pinheiro. El archivador con el acervo de Pierson fue enviado en el mismo camión pero no se avisó del contenido. Resultado: era necesario pintar los muebles y así se hizo. El contenido fue tirado a la basura. Nótese que no había una acta de cesión de bienes (termo de cessão en portugués) de los muebles y del archivo (y para abril de 2000, cuando estuve en ambas instituciones tratando de entender cómo sucedió esa pérdida, todavía no la había), o sea, la transferencia fue informal y no hubo interés de ninguna de las partes en designar a una persona informada del contenido y del valor del acervo que supervisara personalmente la transferencia, lo que obviamente exime al pobre pintor de cualquier culpa, y finca en las instituciones la responsabilidad de lo ocurrido.

Deteniéndonos a pensar en el bote de basura donde fueron depositados, fiel y delicadamente, los millares de fichas de trabajo de campo, nos quedan enormes interrogaciones: ¿a quién sirve esta práctica del olvido?, ¿cómo es posible crear una memoria técnica de las ciencias sociales, del modo de vida de las poblaciones tradicionales brasileñas, e incluso de los proyectos nacionales brasileños, si muchas veces las propias instituciones que deberían preservar este acervo no lo hacen? ${ }^{13}$

Considerando la memoria como un puente entre el pasado y el presente, aunque motivada por el presente, y a

\footnotetext{
${ }^{13}$ Vale la pena recordar y reconocer el trabajo del personal del Acervo Memoria de la CODEVASF que impidió, literalmente, el reciclaje de todo el material referente a la extinta CVSF, donde se encuentran, por ejemplo, los originales del acta del convenio entre la ELSP y la CVSF. A
} 
pesar de la importancia de Pierson en la antropología de Brasil ${ }^{14}$, parece que nuestro autor cayó en el "lado oscuro de la memoria", ya sea por "darwinismo intelectual" o por una "visión hobbesiana de la vida intelectual"15.

Entre las posibles explicaciones de tal ostracismo menciono dos: la primera se refiere a una cuestión metodológica; a saber, la estrecha asociación entre Pierson y los estudios de comunidades. Para Josildeth Consorte, Pierson fue "el principal incentivador de este tipo de estudios entre nosotros" (Consorte, 1996: 71). En segundo lugar, pero ligadas al prejuicio, están las críticas a la manera en que condujo las conclusiones de su estudio sobre las razas en el estado de Bahía (y a la teoría que derivó de ese estudio): subliminalmente, él habría ido a Brasil a procurar una situación racial mejor que la estadounidense (Pierson había realizado un survey en Nashville antes de venir a Brasil).

Acerca de las críticas a la metonimia entre los estudios de comunidades y Pierson, hay algunos aspectos que deben considerarse. El primero es que, de hecho, así como hubo un ciclo de estudios de comunidades en Brasil, también hubo un "ciclo de críticas" y, en esta danza de la teoría, muchos autores y referentes teóricos de Pierson (y él mismo) fueron dejados en el ostracismo ${ }^{16}$, a veces injustamente, pues en relación con sus propios escritos etno-

continuación cito a una de las trabajadoras: "Había una enorme cantidad de cajas en el garage [del edificio de la CODEVASF en Brasilia, Distrito Federal] y nadie sabía lo que era aquello: ¡Ah... es papel viejo, es para reciclar. Un día, otra bibliotecaria y yo fuimos a ver lo que era y nos asustamos: ¡eran todos los documentos de la desaparecida CVSF! Entonces creamos el Acervo Memoria, para impedir que aquello fuera a dar a la basura" (entrevista con la bibliotecaria de la CODEVASF, 1999).

${ }^{14}$ No tiene sentido retomar aquí una lista de artículos y libros editados, traducidos y firmados por él (por su propia cuenta, siete libros y 70 artículos) dado que esto puede encontrarse en la charla entre Pierson y Corrêa (1987: 63-68) y en Lippi Oliveira (1987), donde también aparece una lista de alumnos que él asesoró.

${ }^{15}$ Expresiones utilizadas por Maria Laura Viveiros de Castro Cavalcanti para explicar el no reconocimiento de la importancia de Oracy Nogueira - alumno, amigo y colaborador de Pierson- en la sociología brasileña (véase <www.fiocruz.br/hscience/vol2n2/dep22. html >). Agradezco también a Maria Laura el acceso a algunas de las cartas de Oracy Nogueira dirigidas a Pierson en las que se trata el olvido de ambos.

${ }^{16}$ Basta con ver los autores a quienes él dedica su libro Teoría e Pesquisa em Sociologia (1970 [1945]): "Simmel, Durkheim y Sumner, los pioneros de la sociología científica”. Aunque Durkheim sigue leyéndose bastante y su obra se sigue editando, Simmel pasó mucho tiempo sin leerse en Brasil y Sumner no se ha reeditado en portugués desde 1950. gráficos, si Cruz das Almas es un estudio de comunidad, no podríamos decir lo mismo de O Homem no Vale do São Francisco.

No quiero detenerme en la crítica a las conclusiones de sus estudios sobre la raza pues estoy consciente de que es necesaria una discusión más amplia sobre la influencia de Pierson en sus alumnos para verificar hasta qué punto "los etnógrafos de los países periféricos habían aprendido con sus maestros de los países centrales la teoría del mirar descentrado que presuponía fijar el punto de fuga de esa perspectiva universalista en la cultura occidental europea" (Carvalho, 1999: 5). En el caso del autor de Negros e Brancos na Bahia, incluso una crítica coherente como ésta puede ser descartada: es que entre todos — críticos y defensores - hay unanimidad en ver la obra de Pierson y su actividad docente como el punto de partida de la renovación de los estudios sobre la raza en Brasil ${ }^{17}$. Es decir, no hay manera de negar el papel de Pierson como promotor y fundador de un discurso cuyos desdobles originaron algunas de las más interesantes perspectivas sobre el racismo en Brasil en dos de sus alumnos, amigos, colaboradores y —en algunos momentos- férreos críticos: Oracy Nogueira y Florestan Fernandes $^{18}$.

O sea, hay algo en la crítica que termina por enaltecer a Pierson. Si pensamos que el buen profesor es aquel que desea que sus alumnos vean más lejos que él, esa faceta es reconocida por sus exalumnos, como se desprende del testimonio (2003) de Esdras Borges Costa, profesor jubilado de la Fundación Getúlio Vargas: "si el trabajo de Pierson no floreció en la Escuela Libre de Sociología y Política (ELSP), como él quería, floreció en nosotros, en las instituciones donde trabajamos o en las que creamos". Pero no es sobre este aspecto de la producción de Pierson que versa el presente texto, aunque es relevante la alusión a su papel como director de tesis para entender la importancia del acervo perdido. Volvamos, entonces, a las cuestiones que son objeto de este artículo.

\footnotetext{
${ }^{17}$ Véase Bacelar, 1997; Cavalcanti, 1999 y s. a.; y Nogueira, 1970.

18 Sin contar la importancia que Guerreiro Ramos — otro autor central en el pensamiento sobre el racismo en Brasil-concedió a las clases que tomó con Pierson en su formación intelectual. Véase Lippi Oliveira, 1995.
} 
Por numerosas que sean las críticas, hay algo en lo que nadie cuestiona a Pierson: su capacidad para observar y describir la vida de las comunidades que estudiaba, algo que tiene que ver con el propio rigor de su metodología de observación y que se percibe también en el cuidado con que acometía la tarea de dirigir los trabajos de sus alumnos. Al respecto, es interesante ver el Apéndice II de Pierson (1970) ("Algunas sugerencias metodológicas para el estudio de una comunidad rural"). Originalmente se trataba de una guía de sugerencias de investigación utilizada por los miembros del Proyecto San Francisco con el objetivo de dar uniformidad a las rutinas y los temas, de manera que las investigaciones realizadas por los cinco equipos pudieran ser comparadas y sistematizadas ${ }^{19}$. A Pierson le preocupaba en extremo registrar los dichos y acontecimientos en los términos de los propios nativos ${ }^{20}$, sabedor tal vez de la caducidad de su teoría, pero también de la importancia del retrato fiel, con más razón tratándose de regiones donde había un total desconocimiento de la demografía local ${ }^{21}$ y hasta de lo más elemental de la geografía local ${ }^{22}$. Pierson era consciente de esto y no se

\footnotetext{
${ }^{19}$ Véanse los fólderes 064 y 066 del acervo de Pierson ya citado, donde se encuentra la correspondencia intercambiada entre él y los grupos de investigadores del Proyecto San Francisco, en campo. Es interesante ver cómo cada carta de pronto era respondida con largas listas de sugerencias. Además, cuando sentía que una de las cuestiones planteadas por alguno de los investigadores podría interesar al resto, inmediatamente enviaba copias a los otros equipos para que las ideas circularan continuamente. Hay que recordar que todo esto sucedió antes de la llegada de las fotocopiadoras a Brasil, y que toda esa red se tejía vía correo aéreo, ya que en esa época Brasil era una especie de archipiélago y el transporte interregional se hacía por vía marítima, fluvial o aérea.

${ }^{20}$ Esta práctica impidió la publicación en inglés del libro sobre el Valle de San Francisco, pues había muchísimas expresiones difíciles de traducir que Pierson prefirió conservar en portugués, lo cual dejó bastante insatisfechos a los editores. También por eso, aunque listo desde 1959, el texto en inglés sólo tuvo su versión final hasta 1991. El original de esta obra se encuentra en la colección Pierson de la Universidad de Florida (en línea: < http://web.uflib.ufl.edu/spec/manus cript/guides/Pierson.htm $>$ ).

${ }^{21}$ Véase el prefacio del tomo I de Pierson, 1972, para tener una idea de lo difícil que era obtener datos concretos sobre mortalidad infantil, crecimiento poblacional, etc. Debido a tal carencia, y dado al "hágalo usted mismo", el primer censo confiable de las localidades estudiadas fue realizado por el equipo de Pierson. Hecho que ya había ocurrido antes: como muestra Bacelar (1997), los datos presentados por Pierson (1945) para la región del Recôncavo Baiano todavía son los mejores que hay para la época.

${ }^{22}$ Los únicos datos sobre la longitud del río de que se disponía eran los levantados por Halfeld ien 1860 !
}

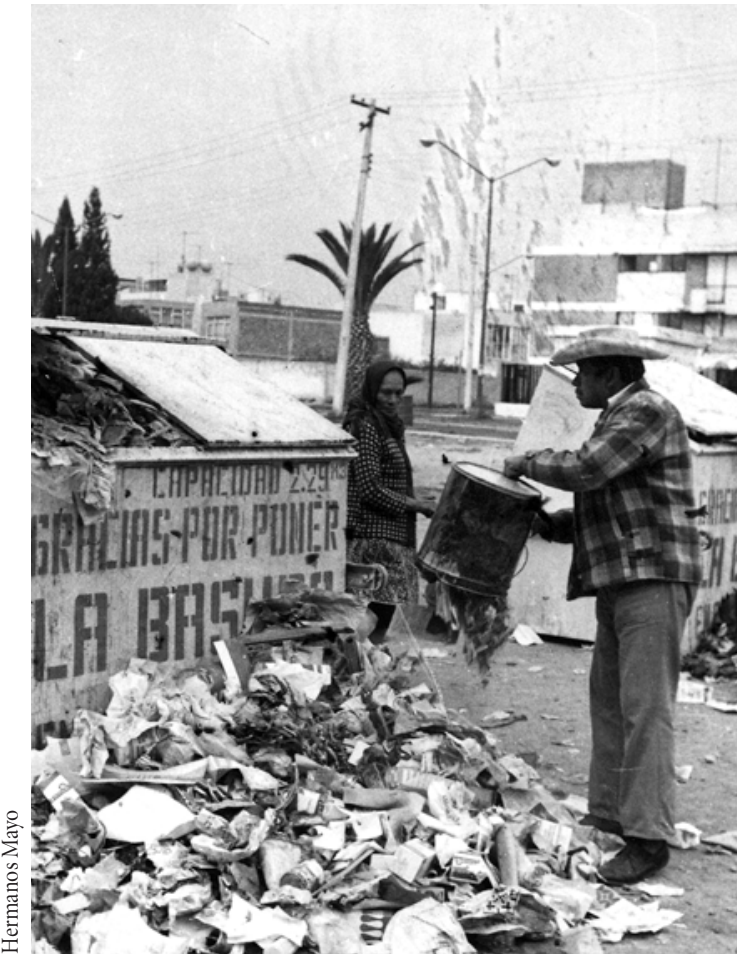

Escenas más recientes de la ciudad, 1971.

conformaba con la indiferencia con la que, hasta cierto punto, había sido tratada su obra, como se desprende de una carta dirigida a Oracy Nogueira, fechada el 25 de octubre de 1972, cuando ya era seguro que se editaría $O$ Homem no Vale do São Francisco, obra monumental que demoró 13 años en publicarse (entre la fecha de entrega del manuscrito y su presentación):

Contando con su comprensión amiga y respeto, tal vez convenga decir aquí de paso que me sentí enormemente preocupado cuando tuve que esperar durante largos años la publicación de esta obra, sabiendo que a cada día que pasaba la información perdía actualidad. Creo que no será difícil entender la "agonía intelectual" por la que pasé.

En aquella época, puedo confesarlo también, francamente, el único consuelo que tenía era saber que, cualquiera que fuera la pérdida de actualidad de dicho material, después de tan larga demora, la obra no carecería de valor histórico pues las investigaciones se habían hecho con cuidado; al contrario, el valor se incrementaría a cada año que pasara ${ }^{23}$.

${ }^{23}$ Carpeta 041 del acervo de Donald Pierson en el Archivo Edgard Leuenroth. 
Dicha preocupación, que varias veces fue calificada de manera simplista de empiricista, en realidad disfrazaba lo que para él era la misión de su vida: "la preparación de futuros investigadores en ciencias sociales a través de investigaciones hechas por ellos mismos, bajo la dirección de personas más experimentadas, desarrollándose, en el proceso, cualquier otra actividad necesaria para su realización" (Pierson, 1987: 42 y Cavalcanti, 1999: 99). En el marco de este proyecto, Pierson formó a 22 investigadores.

Y efectivamente, en este proceso se acumuló una inmensa cantidad de datos primarios sobre el Río San Francisco, material que sólo fue usado en parte en las publicaciones del grupo de investigadores, restando "mucha y mucha cosa inédita”. Esta plétora de información del archivo frente a los textos publicados se da porque un libro es una historia que cuenta el autor. Una historia contada es una elección entre otras posibles, las cuales no salen a la luz en esa ocasión pero pueden aparecer posteriormente si los elementos constitutivos son conservados para, en otro momento, ser organizados bajo otros presupuestos y con otros objetivos. Ésta es una cuestión fundamental cuando se habla de archivo. Para Derrida (1995) la gran importancia del archivo está en el porvenir, en él quedan consignados los futuros posibles. Ése es el violento poder que detentan los archivos (y algo todavía más atemorizante: el que detenta quien detenta el archivo, el arconte del archivo).

Entre el 6 y el 8 de abril del año 2000 intenté ordenar lo que quedó del archivo de Pierson en la Biblioteca Pública Luiz de Bessa, a partir de las "palabras clave" anotadas en el encabezado de cada ficha ${ }^{24}$. Aparentemente, la mayor parte de lo que se rescató pertenece a la misma serie, pues encaja en algunos marcadores que también fueron conservados. Además de este material, se encontró una cantidad razonable de fichas que no encajan en estos marcadores (cerca de 30\%). Lamentablemente, la mayor parte de lo que quedó es material de segunda mano, es decir, son fragmentos tomados de las fuentes bibliográficas consultadas. Aun así, se constata que del material restan-

\footnotetext{
${ }^{24}$ Cada ficha tenía, en los términos de Pierson, de cuatro a seis "palabras clave" (por ejemplo: nombre del informante, fecha, nombre de quien colectó la información, lugar, tema, subtema).
}

te (muchas de las fichas están marcadas con una pequeña "v", tal vez indicando su inclusión en el texto o su revisión) se podría elaborar una interesante tesis sobre el Río San Francisco.

De las pocas fichas elaboradas a partir del material "de primera mano" - los fragmentos ordenados de los diarios de campo- rescatadas, algunas eran muy simples, referidas a situaciones prosaicas, como la narración de las dificultades para encontrar alojamiento digno para el equipo de investigadores; otras eran bastante complejas, como, por ejemplo, la presentación de calendarios agrícolas pormenorizados (semejantes a los que Evans Pritchard elaboró y presentó para los nuer), o las descripciones minuciosas de las embarcaciones usadas en el bajo San Francisco. Entonces, es posible imaginar, soñando por un momento, cuántas historias no podrían todavía contarse con la información contenida en aquellas fichas, que prácticamente no fueron consultadas después de la donación. Sólo hay, al respecto, el registro de tres investigadoras del grupo de Pierson que regresaron a consultar el material ${ }^{25}$, y del equipo de la Fundación João Pinheiro que en 1985 elaboró — en buena medida con la ayuda de este fichero — una bibliografía sobre el Río San Francisco, como se muestra en la presentación de la publicación:

En la elaboración de esta investigación deben ser destacadas dos contribuciones: una es la del doctor Donald Pierson, autor de exhaustivas notas sobre el Valle de San Francisco, además de una bibliografía sobre la región. La Fundación tiene la custodia de ese material y usó ampliamente sus citas (Pimenta, 1985: IX).

Si se hace un apretado resumen cronológico de la relación entre Pierson y el Valle de Río San Francisco, es posible percibir una tremenda tensión por mantenerse vivo y en la memoria: en 1950, Pierson hace un viaje por todo el Valle del Río para seleccionar las ciudades que serían estudiadas; en 1951 firma el convenio con la CVSF para realizar la investigación colectiva; en 1952, los equipos hacen trabajo de campo; a lo largo de la década de 1950 y prin-

\footnotetext{
${ }^{25}$ Se trata de Márcia Alves de Souza, Cecília Maria Di Lascio y Haydée Guanais Dourado (Pierson en Corrêa, 1987: 110).
} 


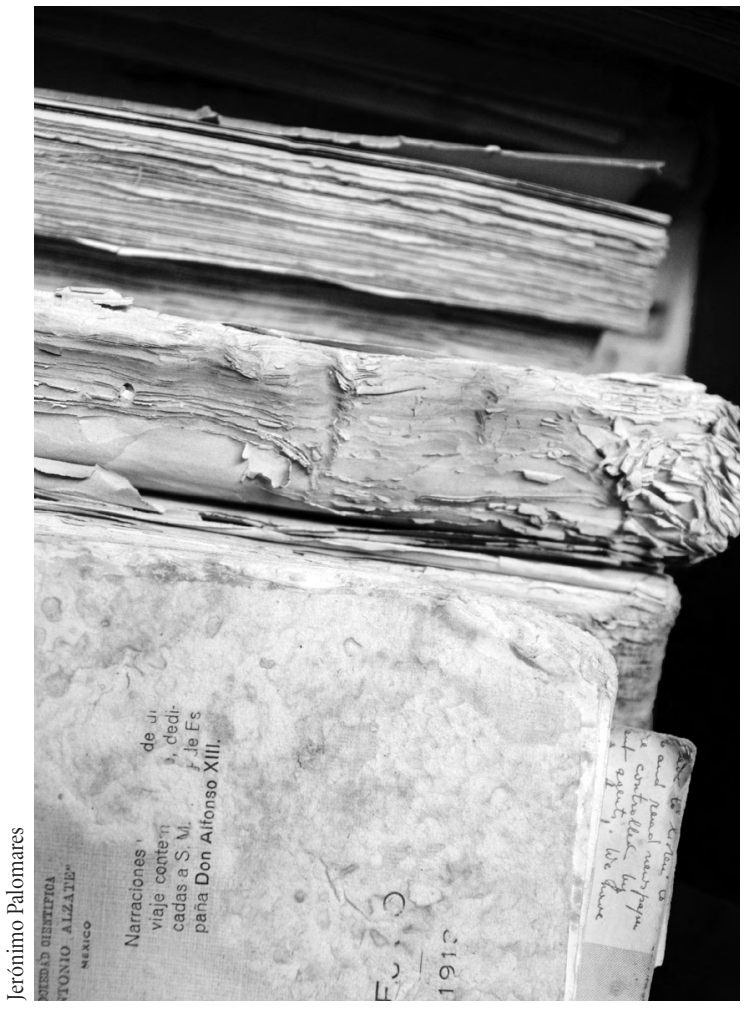

Biblioteca “Rafael García Granados”, 2010.

cipio de la siguiente, se publican decenas de artículos de los participantes del proyecto (como ya se mencionó, buena parte en la revista Sociología) y las monografías de los jefes de equipo (publicadas por la CVSF); en 1959 queda listo el libro síntesis de Pierson. Ese periodo marca el ápice de la productividad de Pierson, pues a mediados de la década de 1950 sale de Brasil por motivos de salud y regresa a Estados Unidos, sin lograr recuperarse del todo.

El libro O Homem no Vale do São Francisco quedó listo en 1959, "durmió" durante 13 años en diferentes cajones de comisiones y ministerios, dándose por perdido en más de una ocasión, hasta que fue publicado en 1972. En esa etapa Pierson mantiene una vasta correspondencia con sus colegas de la ELSP —en esos días pasaba por una severa crisis- y con conocidos suyos del Congreso Nacional brasileño y de la CVSF/SUVALE, con el objetivo de mantener al grupo de la ELSP y tratando de dar segui- miento a las publicaciones. Con el temor de morir y el riesgo de que el material del trabajo de campo se perdiera, Pierson dona el archivo a la Fundación João Pinheiro, que lo recibe en 1983.

La tensión que permea gran parte de la correspondencia conservada en el Archivo Edgard Leuenroth también se evidencia en las entrevistas que hice a varias personas que de alguna manera trabajaron con él (como Mireya Suárez, Mariza Corrêa y Sebastião Vila Nova). Tanto para Suárez (de quien fue asesor en la Escuela Nacional de Antropología e Historia, México) como para Corrêa, Pierson vivió con el sentimiento de que las ciencias sociales en Brasil eran malagradecidas. Vila Nova señala que el desinterés es de tal magnitud que la biografía de Pierson que él escribió fue publicada en Portugal (1998), pues ninguna editorial brasileña se mostró interesada en ella.

Finalmente, Pierson falleció en 1995 y, sólo cuatro años después, el material bruto de lo que él llamó su "más ambicioso proyecto" fue tirado a la basura. Hay una frase que no está en el mensaje original de la Fundación João Pinheiro citado en este texto, pero que empuja como queriendo ser enunciada, de manera que tomé la libertad de reescribir el párrafo agregando lo que en mi opinión faltaría, aunque entre corchetes:

Todas las fichas fueron tiradas a la basura $[\ldots]$ para nosotros fue motivo de gran insatisfacción y frustración pues sabíamos de la enorme importancia del archivo, nos sentimos culpables de la pérdida y de la falla, sin saber dónde nos habíamos equivocado. [Ese archivo nunca fue investigado] y por increíble que parezca, desde entonces varias personas nos han buscado para consultar tales fichas.

Desde mi punto de vista, la frase faltante revelaría una vía para la interpretación de lo sucedido, pues pone en jaque la conciencia de la importancia del archivo por parte de las propias instituciones encargadas de custodiarlo. Aunque la frase no apareciera en el correo electrónico, yo la escuché más de una vez cuando estuve en las dos instituciones verificando el material que había quedado. Esta frase siempre venía acompañada de otra aún más non sense - “ $[. .$.$] pero, ¿̇no estás de acuerdo conmi-$ go en que cuando un autor publica un texto él ya usó todo lo que era interesante de sus notas?" - que era uti- 
lizada para minimizar el impacto de la destrucción del $\operatorname{archivo}^{26}$.

Es necesaria aquí una digresión. Este texto trata de la destrucción de un archivo, pero la historia reciente de Brasil es permanentemente construida a partir de este tipo de supresión de rastros, lo que sitúa el "caso del archivo de Pierson" como sólo uno de los elementos de una vasta estructura de silenciamiento de las voces de los vencidos. Baste recordar que la República Brasileña, declarada en 1889, se inaugura con la orden dada por Ruy Barbosa de destruir los archivos de la esclavitud (1890) y con la masacre de Canudos (1897), en la que miles de campesinos fueron asesinados por el ejército brasileño. Además, la manera en que se han hecho los arreglos políticos en Brasil siempre ha privilegiado el desvanecimiento de la memoria, un ingrediente más de la vulnerabilidad de los acervos en el país. Un ejemplo de esta situación puede ser la inexistencia de artículos semejantes a éste que relaten e interpreten la destrucción de la biblioteca del Centro Latinoamericano de Investigación en Ciencias Sociales, ocurrida durante el último periodo dictatorial.

116 Como dijera Walter Benjamin, el pasado siempre está en riesgo.

Lo descrito hasta ahora lleva a invertir la famosa frase de Benjamin (2008): "todo documento de barbarie también es un documento de cultura”. Porque la creación de la autonombrada "nación cordial" se hace gracias a la supresión de las huellas de la violencia perpetrada en contra de los "subalternos" y del silenciamiento de los discursos alternativos sobre los procesos históricos. El caso que nos ocupa, la destrucción del acervo de Pierson, sería un excelente ejemplo (en su lado absurdo) de la construcción del pasado vacío y homogéneo de la nación (Anderson, 1993; Jameson, 1981; Bhabha, 2002). Desafortunadamente, es posible pensar un acto de destrucción por pura negligencia, como en este caso, como una forma de borrar otras versiones potenciales del pasado, o la posibilidad de respuesta de un investigador que muchas veces fue criticado más de lo que correspondería.

\footnotetext{
${ }^{26}$ Este tipo de cuestiones ya fue planteado y no creo que sea necesario volver a tal discusión.
}

Pero también tenemos, en éste y en otros casos ${ }^{27}$, ejemplos de cómo se instituyen y se "desinstituyen" las instituciones en Brasil. Y en este punto aflora otra cuestión que al parecer también tuvo que ver con lo sucedido: la salida del acervo de Pierson de la Fundación João Pinheiro obedeció, al parecer, a los propósitos actuales de dicha fundación de trabajar con políticas públicas de Minas $\mathrm{Gerais}^{28}$, y el Río São Francisco, con sus más de 2600 km, excede con mucho ese estado. Por otro lado, exactamente por las mismas razones, este acervo no encajaba en el perfil de la colección a la que fue donado: la Colección Mineiriana de la Biblioteca Pública que, como su nombre lo dice, también estaba centrada en Minas Gerais. Sólo tal coincidencia podría explicar el extraño hecho de que el acervo pudiera salir de un lugar y no necesitara llegar a otro, pues fue eso precisamente lo que sucedió: no hubo acta de cesión, no se supervisó el traslado y, en apariencia, tampoco hubo la intención de saber si la persona responsable de la recepción del archivo estaría en la Biblioteca (la persona que estaba "al corriente de la donación [...] estaba de vacaciones”).

Con esto llegamos al final de este artículo informativo que deja todavía algunas preguntas resonando: la autoridad del arconte sobre el archivo ies —o puede o debe ser- - tan grande que éste tenga la facultad para destruirlo? En la Reunión Brasileña de Antropología del año 2008, Lívio Sansoni presentó, en el grupo de trabajo “Archivos e historias de la antropología brasileña: tradiciones visibles e invisibles", el proyecto de lo que él llamó un "anti-museo de la antropología de las relaciones raciales en Brasil”, que estaba implementando en el Centro de Estudios Afroorientales de la Universidad Federal de Bahia

\footnotetext{
${ }^{27}$ Como la afortunada casualidad de la preservación del acervo de la CVSF, ya citada.

${ }^{28}$ En el sitio de la Fundación João Pinheiro [<www.fjp.gov.br/fjpinst.html>] queda muy claro: "La Fundación João Pinheiro es una entidad del gobierno de Minas Gerais dedicada a la realización de proyectos de investigación aplicada, consultorías, formación de recursos humanos y acciones de apoyo técnico al Sistema Estatal de Planeación y demás sistemas operativos de Minas [Gerais], en las áreas de la administración pública y privada, la economía, los estudios históricos, culturales, sociales y políticos" (subrayado mío, consultado en abril de 2002).
} 
(CEAO/UFBA). Su propuesta consistía exactamente en reducir el poder de consignación del "propietario" del archivo mediante la creación de acervos virtuales (algo cada vez más fácil de hacer) de antropólogos brasileños, o que trabajaran en Brasil sobre este tema. A lo largo de su intervención, Sansoni presentó diversas copias digitales de fotos, cartas, notas y fonogramas de autores como Frazier, Pierson, Herskovits, entre otros, y se discutió la posibilidad de que la Asociación Brasileña de Antropología tuviera un papel de facilitador en la intermediación — para la digitalización y divulgación — entre las instituciones interesadas y los "propietarios" (en el caso de los archivos privados en manos de los parientes) o depositarios, es decir, hasta cierto punto, una adaptación para la era del internet de la moción que yo mismo presenté a la Asociación Brasileña de Antropología en el año 2000 (véase Anexo). A final de cuentas, las notas originadas en el inmenso Proyecto San Francisco fueron a dar a la basura sólo cuatro años después de la muerte de Pierson. Cabe entonces preguntarnos, más allá de toda ilusión biográfica y bibliográfica, ¿cuántas muertes pueden ser imputadas a un investigador? Tal vez aquí tengamos un proyecto para evitar otras muertes futuras.

\section{ANEXO}

\section{Moción de repudio a la destrucción del archivo del profesor Donald Pierson, a la falta de cuidado a los acervos de los archivos en general y sugerencias de providencias a tomar*}

Excelentísimos señores Presidente de la República, Secretario de Cultura, Secretario de Educación:

En diciembre de 1998, mediante una desafortunada operación de transferencia — en la que estuvieron involucradas la Fundación João Pinheiro y la Biblioteca Pública de

\footnotetext{
* Moción aprobada en la Asamblea General de la Asociación Brasileña de Antropología llevada a cabo el 19 de julio de 2000. Suscrita por el doctor Antonio Augusto Arantes, la doctora Marisa Veloso, el doctor Gilberto Velho y el maestro Roberto Lima.
}

Belo Horizonte- se tiró a la basura cerca de $80 \%$ del material de campo recolectado por Donald Pierson y 22 colaboradores, referente al "Proyecto São Francisco" que, en la década de los cincuenta, involucró institucionalmente a la Escuela Libre de Sociología y Política, a la Comisión del Valle del Río São Francisco y al Smithsonian Institute, y que fue, sin duda, uno de los más ambiciosos proyectos llevados a cabo en Brasil, en el ámbito de las ciencias sociales.

Desgraciadamente, ésta es una práctica recurrente en nuestro país, basta recordar la biblioteca del Centro Latinoamericano en Río de Janeiro, cuyo material fue totalmente transformado en papel para reciclar. Es necesario recordar que los acervos históricos están protegidos por la Constitución Federal, cuyo artículo 23, referente a la conservación del patrimonio público, señala:

Es competencia común de la Unión, de los Estados, del Distrito Federal y de los Municipios:

I. Velar por el respeto a la Constitución, las leyes y las instituciones democráticas y conservar el patrimonio público; [...] III. Proteger los documentos, las obras y otros bienes de valor histórico, artístico y cultural, los monumentos, los paisajes naturales notables y los sitios arqueológicos; IV. Impedir la evasión, la destrucción y la alteración de obras de arte y de otros bienes de valor histórico, artístico o cultural; V. Proporcionar los medios de acceso a la cultura, a la educación y a la ciencia;

En este caso, la cuestión que se plantea es: ¿qué hacer cuando las instituciones que deberían ser las responsables de la conservación de archivos históricos en lugar de eso los destruyen? Muchas veces esas expurgaciones son motivadas por cambios en los objetivos de las fundaciones y empresas que tienen la custodia de los archivos, pero eso no se puede traducir en la simple eliminación del acervo. Actualmente existen en Brasil archivos idóneos que pueden ser contactados para la transferencia de acervos. Es importante subrayar que debe haber más interés en que estos traslados se den de manera legal y segura, para que no se repitan acontecimientos desafortunados como la pérdida de valiosos acervos bibliográficos. En vista de lo anterior, hacemos dos propuestas:

En lo que se refiere al archivo perdido de Donald Pierson, en particular, que las dos instituciones involucradas 
se comprometan, como una medida compensatoria, a localizar a los investigadores que sigan vivos del grupo de Pierson con la intención de resguardar sus archivos personales (Obs. Eventualmente estos archivos personales podrán ser custodiados por otras instituciones, en caso de que estén más seguros); desde el punto de vista general, que se cree un "directorio temático de archivos", a nivel nacional, que permita, a las instituciones que tengan archivos de valor histórico, donar con seguridad sus documentos a instituciones en las cuales éstos sean resguardados y puedan ser consultados.

\section{Bibliografía}

Anderson, Benedict, 1993, Comunidades imaginadas, Fondo de Cultura Económica, México.

Bacelar, Jeferson, 1997, "Donald Pierson e os brancos e negros na Bahia”, Horizontes Antropológicos, año 3, núm. 7, pp. 129-143.

Bhabha, Homi, 2002, El lugar de la cultura, Manantial, Buenos Aires.

118 Benjamin, Walter, 2008, Tesis sobre la historia y otros fragmentos, Universidad Autónoma de la Ciudad de México, México.

Carvalho, José Jorge, 1990, O Olhar Etnográfico e a Voz Subalterna, Série Antropológica 261, Universidad de Brasilia, Brasilia.

Cavalcanti, Maria Laura V. C., 1999, "Preconcepto de marca: etnografia e relações sociais”, Tempo Social, núm 11, vol. 1.

— - s. a., "Oracy Nogueira: esboço de uma trajetória intelectual", en línea: <www.fiocruz.br/hscience/vol2n2/dep22.ht$\mathrm{ml}>$, consultado el 12 de enero de 2002.

Consorte, Josildeth Gomes, 1996, "Os Estudos de Comunidades no Brasil: Uma Viagem no Tempo", en Maria Isabel Faleiros (org.), Humanismo e Compromisso: Ensaios Sobre Octavio Ianni, un esp, São Paulo.

Corrêa, Mariza, 1987, História da Antropologia do Brasil (19301960), Vértice, Campinas.

Dantas, Audálio, 1972, "O país do São Francisco", Realidade, vol. 6, núm. 72, pp. 34-102.

Derrida, Jacques, 1997, Mal de archivo. Una impresión freudiana, Trotta, Madrid (accesible en línea: <http://xoomer.
virgili.it/martinm/PUG/Malderarchivo.pdf $>$, consultado el 5 de mayo de 2009).

Jameson, Fredric, 1981, The Political Unconscious: Narrative as a Socially Symbolic Act, Routledge, Londres.

Nogueira, Oracy, 1970, "Donald Pierson e o desenvolvimento da sociologia no Brasil”, Universitas, núms. 6-7, pp. 331-342.

Lippi Oliveira, Lucia, 1987, "Donald Pierson e a Sociologia no Brasil”, BIB, núm. 23, pp. 35-48.

— 1995, A sociologia do Guerreiro, Editora de la Universidad Federal de Río de Janeiro, Río de Janeiro.

Peltier, Paulo y Donald Pierson, 1951, "Convênio para a realização de estudos de caráter ecológico e sociológico", Libro Acta núm. 1 de la Compañía del Valle de San Francisco (10 de diciembre de 1949-23 de septiembre de 1952), Acervo Memoria de la Compañía de Desarrollo de los Valles de San Francisco y del Parnaíba, Brasília, pp. 85-89, manuscrito.

Pierson, Donald, 1945, Bancos e pretos na Bahia: estudo de contato racial, Compañía Editora Nacional, São Paulo.

__ 1987, "Algumas atividades no Brasil em prol da Antropología e outras ciências", en Mariza Corrêa, História da Antropología no Brasil (1930-1960: Testemunhos, Vértice, Campinas.

—_, 1970 [1945], Teoria e Pesquisa em Sociologia, Melhoramentos, São Paulo.

—_ 1972, O Homem no Vale do São Francisco, 3 tomos, SUVALE/ Ministerio del Interior, Río de Janeiro.

Pimenta, Aloísio, 1985, Prefácio, en Fundação João Pinheiro, Vale do São Francisco: bibliografia, Fundación João Pinheiro, Belo Horizonte.

Vila Nova, Sebastião, 1998, Donald Pierson e a Escola de Chicago na sociologia brasileira: entre humanistas e mesiánicos, Vega, Lisboa.

\section{Archivos y página electrónica consultados}

Archivo Edgard Leuenroth, Universidad de Campinas.

Acervo Memoria de la Compañía de Desarrollo de los Valles de San Francisco y del Parnaíba (CODEVASF).

Archivo de Pierson en la Biblioteca Pública Luiz de Bessa de Belo Horizonte, Minas Gerais.

Donald Pierson Papers, Special and Area Studies Collections, George A. Smathers Libraries, University of Florida, Gainesville, Florida. En línea: <http://web.uflib.ufl.edu/spec/manuscript/ guides/Pierson.htm>, consultado el 3 de marzo de 2009. 